Results The national survey was completed by 166 students representing 22 UK medical schools. Students reported limited interest, knowledge and exposure to oncology, lack of confidence in skills, and teaching dissatisfaction. Oncology was perceived as a challenging specialty (mean Likert score: $4.5 / 5 \pm 0.7$ ), yet most students estimate receiving only $1-2$ weeks of dedicated oncology teaching. The national symposium generated a statically significant increase in students' interest $(p=0.0012)$, knowledge, and confidence in skills surrounding oncology $(\mathrm{p}<0.0001)$, improving students' perceived ability to cope with the emotional challenges in this field $(\mathrm{p}=0.0278)$.

Conclusion Students' unimpressive views towards oncology alongside their teaching dissatisfaction underpin the need to reform current undergraduate oncology curricula. Increasing medical student oncology exposure by proposing outcomebased guidelines and adopting a standardised undergraduate oncology curriculum should be the foremost priority in inspiring future oncologists to ensure excellent cancer patient care.

\section{MENTORING MEDICAL STUDENTS TOWARDS ONCOLOGY: RESULTS FROM A PILOT MULTI- INSTITUTIONAL MENTORSHIP PROGRAMME}

Kathrine S Rallis, Anna Maria Wozniak, Sara Hui, Adam Stammer, Cigdem Cinar, Min Sun, Taylor Fulton-Ward, Alison A Clarke, Savvas Papagrigoriadis, Apostolos Papalois, Michail Sideris. Barts Cancer Institute, Queen Mary University of London; Barts and The London School of Medicine and Dentistry, Queen Mary University of London; Birmingham Medical School, University of Birmingham; Guy's King's and St Thomas' School of Medicine, King's College London; International Society for Pelvic Surgery, Athens, Greece; Experimental Educational and Research Centre ELPEN, Athens, Greece; Women's Health Research Unit, Queen Mary University of London

\subsection{6/spcare-2021-PCC.53}

Background The mounting global cancer burden has generated an increasing demand for oncologists to join the workforce. Yet, students report limited oncology exposure in undergraduate medical curricula, while undergraduate oncology mentorships remain underutilised. We established an undergraduate oncology society-led mentorship programme aimed at medical students across several United Kingdom universities to increase medical student oncology exposure.

Methods We electronically recruited and paired oncologist mentors and medical student mentees and distributed a dedicated questionnaire (pre and post-mentorship) to compare mentees' self-reported cancer specialty knowledge and oncology career motivation after undertaking a 6-week mentorship. We also determined students' interest across specialties and subspecialties and measured mentor availability via percentage programme uptake. Statistical analysis included univariate inferential tests on SPSS software.

Results Twenty-nine (23.4\%) of 124 oncology specialists agreed to become mentors. The mentorship was completed by 30 students across 3 medical schools: 16 (53.3\%) Barts, 10 (33.3\%) Birmingham, and 4 (13.3\%) King's; 11 (36.7\%) mentored by medical oncologists, $10(33.3 \%)$ by clinical/radiation oncologists, and 9 (30\%) by surgical oncologists. The mentorship generated a statically significant increase in students' knowledge of the multidisciplinary team $(p<0.001)$ as well as the role of medical $(p<0.001)$, surgical $(p=0.006)$, and clinical oncologists $(p<0.001)$ and their involvement in academia/ research $(p=0.001)$. Mentees' interest in oncology remained unchanged. Further feedback demonstrated that $93.3 \%$ of mentees believed that the mentorship scheme made them a better medical student or a future doctor. Additionally, 96.7\% of students reported that they would chose to take part in the programme again.

Conclusion Undergraduate oncology mentoring is an effective educational, networking and motivational tool for medical students. Student societies are a valuable asset in cultivating medical student oncology interest by connecting students to faculty and increasing mentor accessibility. Further research should focus on developing an optimal mentorship structure and evaluating long-term outcomes of such educational initiatives.

\section{ENABLING CONNECTIONS: USING PROJECT ECHO (EXTENSION OF COMMUNITY HEALTHCARE OUTCOMES) IN PALLIATIVE CARE EDUCATION}

Anne Kelly, Jane Andrew, Claire Douglas. NHS Tayside Palliative Care

\subsection{6/spcare-2021-PCC.54}

Introduction The need for palliative and end of life care is increasing in Scotland. Workforce development is required to support practitioners to be knowledgeable, skilled and confident in providing palliative care. Project ECHO is a validated, technology-enabled, educational approach used globally to connect networks of learners and form communities of practice. We were the first NHS Scotland palliative care service to use this model. The aims of our pilot project were

- To establish, deliver and evaluate ECHO groups as supportive communities of practice.

- To connect participants with tele-mentoring and support from Specialist Palliative Care services

Methods Project ECHO groups were established with community hospitals and care homes. Project ECHO uses a hub and spoke model. Each session comprises a formal teaching component, a case presentation brought by participants and facilitated case-based discussion. Evaluation was guided by a Logic Model aligned to Kirkpatrick's model 6 for educational evaluation. Mixed methods were used to gather evaluation data, including Survey Monkey, focus groups and reflective feedback.

Results All ECHO sessions were highly rated in terms of quality and utility. All participants reported increases in knowledge, skills and confidence. Participants shared reflective pieces highlighting integration of learning into practice and examples of improved patient care. Additional gains were through the building of relationships, greater understanding of other's perspectives and truly learning from and with each other. One year on, all Community Hospital sites remain engaged and their network has been extended. There is growing interest in Project ECHO within care homes.

Conclusions ECHO groups are of relevance and value to members. Project ECHO creates a rich educational environment which can support improved outcomes for patients in our communities. Virtual delivery provides significant savings and enabled the provision of ongoing education and support through Covid-19. 